\title{
Mortalidade pós-fogo em espécies lenhosas de campo sujo submetido a três queimadas prescritas anuais
}

\author{
Marcelo Brilhante de Medeiros ${ }^{1,3}$ e Heloísa Sinátora Miranda ${ }^{2}$
}

Recebido em 28/05/2004. Aceito em 05/12/2004

\begin{abstract}
RESUMO - (Mortalidade pós-fogo em espécies lenhosas de campo sujo submetido a três queimadas prescritas anuais). O fogo é um dos principais agentes de modificação da estrutura e composição das comunidades vegetais em ecossistemas savânicos. Neste estudo, foram avaliados os efeitos do fogo sobre as taxas de mortalidade de indivíduos, mortalidade da parte aérea, considerando os indivíduos que apresentaram destruição do caule, com rebrotas basais e/ou subterrâneas, e número de caules destruídos dos indivíduos de plantas lenhosas, incluindo a soma dos indivíduos que sofreram mortalidade da parte aérea e os indivíduos mortos; estes últimos não apresentaram nenhum tipo de rebrota, sendo eliminados da área. Não foi considerado o recrutamento de indivíduos. Foram realizadas queimadas prescritas anuais, nos anos de 1998, 1999 e 2000, em campo sujo, na Reserva Ecológica do IBGE, Brasília, DF. Em área de 2.500 m², foram inventariadas todas as plantas do estrato arbustivo-arbóreo, com diâmetro igual ou superior a $2 \mathrm{~cm}$ a $30 \mathrm{~cm}$ do nível do solo. Os dados foram analisados com o teste $\chi^{2}(\mathrm{p}<0,05)$. O inventário totalizou 39 espécies, com total de 636 indivíduos. As taxas de mortalidade para as queimadas anuais foram de 22,5, 7,9 e 11,5\%, após as queimadas de 1998, 1999 e 2000, totalizando redução de cerca de $37 \%$ no número de indivíduos vivos. A mortalidade afetou principalmente os indivíduos de menor porte, entre 1 e $2 \mathrm{~m}$ alt. e entre 2 e $3 \mathrm{~cm}$ diâm. Após as três queimadas, os indivíduos mortos adicionados aos que sofreram morte da parte aérea representaram cerca de $73 \%$ do total.
\end{abstract}

Palavras-chave: efeitos do fogo, Cerrado, plantas lenhosas, mortalidade de plantas, campo sujo

\begin{abstract}
Mortality of woody species in a campo sujo after three prescribed annual fires). Fire is one of the agents with most significant effect on the structure and composition of plant communities in savanna ecosystems. This study evaluated the effects of fire on the mortality rates of individual plants, individuals with aerial mortality and basal or underground sprouts (top kill), and the number of destroyed stems which include aerial mortality and dead individuals. The annual prescribed fires were performed in 1998, 1999 and 2000, in a campo sujo area at the Reserva Ecológica do IBGE (Brasília, DF, Brazil). In a 2,500 $\mathrm{m}^{2}$ area all plants of woody layer with diameter equal or greater than $2 \mathrm{~cm}$ at $30 \mathrm{~cm}$ from soil level were surveyed. The data were evaluated with chi-square test $(\mathrm{p}<0,05)$. The survey counted 39 species and 636 individuals. The mortality rates for annual fires were $22.5 \%$ (1998), 7.9\% (1999) and 11.5\% (2000), reducing by $37 \%$ the number of live individuals in the area. Mortality impacted mainly the small individuals - height between 1 and $2 \mathrm{~m}$ high, and 2 and $3 \mathrm{~cm}$ diam. After the three burnings, the dead individuals and those with aerial mortality represented $73 \%$ of total individuals.
\end{abstract}

Key words: fire effects, Cerrado, woody plants, plant mortality, savanna

\section{Introdução}

A ocorrência de queimadas e incêndios, característica dos ecossistemas savânicos, provoca mudanças na florística e na estrutura da vegetação. Pode também ocorrer diminuição de densidade de árvores e arbustos, favorecendo o estabelecimento de certas espécies e provocando eliminação de espécies sensíveis (Frost \& Robertson 1987; Agee 1993; Medina \& Huber 1994). Também ocorrem mudanças nas taxas de crescimento, sucesso reprodutivo (Frost \& Robertson 1987; Hoffmann 1999) e no estabelecimento de plântulas (Bond \& Wilgen 1996; Franco et al. 1996). $\mathrm{O}$ fogo pode reduzir a biomassa vegetal e a serapilheira, alterando os fluxos de energia, nutrientes e água (Frost \& Robertson 1987; Medina \& Silva 1990). A sobrevivência de organismos ao fogo é determinada por suas características anatômicas, fisiológicas e comportamentais, além das características ambientais pós-fogo. As modificações em populações e comunidades dependerão fundamentalmente destas características individuais (Bond \& Wilgen 1996).

A vegetação do Cerrado apresenta características que reforçam a idéia de estratégias adaptativas da

\footnotetext{
' Embrapa Recursos Genéticos e Biotecnologia, Parque Estação Biológica, W3 Norte s/n, C. Postal 02372, CEP 70770-900, Brasília, DF, Brasil

2 Universidade de Brasília, Instituto de Biologia, Departamento de Ecologia, Asa Norte, Campus Universitário Darcy Ribeiro, CEP 70910-900, Brasília, DF, Brasil

3 Autor para correspondência: medeiros@cenargen.embrapa.br
} 
vegetação ao fogo, como forte suberização do tronco e dos galhos, permitindo certo grau de isolamento térmico dos tecidos internos, mesmo em temperaturas elevadas (Coutinho 1990; Rocha e Silva \& Miranda 1996), ocorrência de frutos com capacidade de proteção das sementes (Landim \& Hay 1995), proteção de gemas apicais de algumas espécies por meio de catáfilos (Coutinho 1990) e elevada capacidade de rebrota da copa, de rizomas, caule, raiz e outras estruturas subterrâneas (Souza \& Soares 1983; Coutinho 1990).

Entre os efeitos adversos de queimadas freqüentes para a flora lenhosa, já foi observada a diminuição da densidade arbórea (Souza \& Soares 1983; Sato et al. 1998), sendo que Silva et al. (1996) e Sato et al. (1998) verificaram elevadas taxas de mortalidade em campo sujo e cerrado sensu stricto após queimadas prescritas. $\mathrm{O}$ fogo pode ser fator de mortalidade importante para plântulas no primeiro ano de vida (Franco et al. 1996; Hoffmann 1998) e limitar o crescimento populacional das espécies devido ao impacto no esforço reprodutivo em conseqüência da mortalidade da parte aérea, também conhecida como "top kill" (Hoffmann \& Solbrig 2003).

Este estudo teve por objetivo avaliar os efeitos do fogo sobre as taxas de mortalidade dos indivíduos lenhosos e a conseqüente alteração na estrutura da vegetação, após três queimadas anuais prescritas em uma área de campo sujo que se encontrava protegida do fogo por 23 anos.

\section{Material e métodos}

Área de estudo - O trabalho foi desenvolvido na Reserva Ecológica do Instituto Brasileiro de Geografia e Estatística, IBGE, situada a $35 \mathrm{~km}$ ao sul de Brasília, DF, Brasil. A área caracteriza-se como campo sujo, o qual é definido como uma forma savânica mais rala do Cerrado, tendo o estrato lenhoso cobertura maior do que zero e menor de 10\%; os indivíduos desta camada podem distribuir-se individualmente ou em grupos (Eiten 1994). A área de 1 ha do projeto esteve protegida do fogo por 23 anos, e em 1998 teve demarcada no seu centro uma parcela de $2.500 \mathrm{~m}^{2}$ para a realização dos inventários antes e após as queimadas prescritas. As queimadas foram realizadas nos anos de 1998, $1999 \mathrm{e}$ 2000, na primeira quinzena do mês de agosto, estação seca, quando ocorre a maioria dos incêndios em áreas de Cerrado (Coutinho 1990). As queimadas foram realizadas sempre a favor do vento e com o auxílio da brigada de combate aos incêndios do IBGE.
Inventário da vegetação - Em 1998, antes da primeira queimada, foram inventariados, na área de $2.500 \mathrm{~m}^{2}$, todos os indivíduos do estrato arbustivo-arbóreo, com diâmetro igual ou superior a $2 \mathrm{~cm}$, a $30 \mathrm{~cm}$ do nível do solo. Esses indivíduos foram marcados e a sua altura, diâmetro e espécie foram determinados. Por convenção, foi amostrado o número de caules para cada indivíduo e, quando distantes em mais de $30 \mathrm{~cm}$, foram considerados indivíduos diferentes. Esta consideração deve-se à extensão de estruturas subterrâneas em plantas do Cerrado, as quais podem ligar caules próximos ou distantes de um mesmo indivíduo (Rawitscher et al. 1943). Quando não foi possível a mensuração do diâmetro a $30 \mathrm{~cm}$, caso os caules se separassem em uma altura inferior a esta medida, era considerada a altura de $25 \mathrm{~cm}$, ou menos, caso necessário, em reduções contínuas de $5 \mathrm{~cm}$. Nos meses de junho e julho, ou seja, antes das queimadas, nos anos de 1999, 2000 e 2001, o inventário foi repetido nos mesmos indivíduos amostrados em 1998, sendo o inventário inicial da vegetação a amostra controle do estudo. Neste estudo não foi considerado o recrutamento de indivíduos. Nos inventários de junho e julho, antes das queimadas, além dos indivíduos mortos, também foram contados os indivíduos que apresentaram morte total da parte aérea.

Os indivíduos considerados mortos, com destruição total do caule, diferentemente dos indivíduos com mortalidade aérea, não apresentavam nenhum tipo de rebrota, sendo totalmente eliminados na área.

Dessa forma, o cálculo da taxa de mortalidade de indivíduos $(M t)$ não considerou os indivíduos com mortalidade aérea, uma vez que estes indivíduos sobreviveram às queimadas, e foi calculada de acordo com a seguinte equação:

$$
M t=\frac{M .100 \%}{V}
$$

onde $M$ é o número de indivíduos mortos e $V$ é o número de indivíduos vivos, sem considerar o recrutamento de novos indivíduos.

A percentagem de caules destruídos $(C D)$ foi calculada de acordo com a equação descrita por A.E. Ramos (dados não publicados) e modificada por E.P. Rocha e Silva (dados não publicados):

$$
C D=\frac{T k+M}{V} \cdot 100 \%
$$

onde $T k$ é o número de indivíduos com mortalidade aérea, $M$ é o número de indivíduos mortos e $V$ é o número de indivíduos vivos antes da queima, sem 
considerar recrutamento. Os indivíduos considerados com mortalidade aérea foram analisados de acordo com o conceito de Whelan (1995) para "top kill". Estes indivíduos apresentavam, após as queimadas, o caule totalmente destruído, com rebrota basal e/ou subterrânea.

As comparações entre os dados de estrutura da vegetação e mortalidade foram analisadas com o teste de $\chi^{2}(\mathrm{p}<0,05)$ (Sokal \& Rohlf 1997), utilizando-se o programa Statistica (2000).

\section{Resultados e discussão}

Estrutura da vegetação antes da queimada - O número de indivíduos e a freqüência relativa das espécies lenhosas encontradas na área de estudo antes da primeira queimada prescrita em 1998 são apresentados na Tab. 1. Este inventário totalizou 39 espécies do total de 636 indivíduos. Dentre estas espécies, 13 apresentaram mais de 10 indivíduos: Acosmium dasycarpum, Byrsonima verbascifolia, Connarus fulvus, Davilla elliptica, Dimorphandra mollis, Eremanthus goyazensis, Erythroxylum suberosum, Kielmeyera coriacea, Ouratea hexasperma, Roupala montana, Rourea induta, Styrax ferrugineus e Vellozia flavicans. Estas espécies representaram $87 \%$ dos indivíduos inventariados. Destacaram-se, pelo elevado número de indivíduos, Davilla elliptica e Roupala montana, com 92 e 123 indivíduos, respectivamente, representando $34 \%$ do total de indivíduos inventariados. Nove espécies apresentaram apenas um indivíduo.

Os indivíduos inventariados apresentaram altura entre 0,3 e 7,0 m. Os indivíduos com menos de $1 \mathrm{~m}$ representaram $19,8 \%$ do total (126 indivíduos) e os com altura entre 1,0 e 2,0 m, 71,9\% do total de indivíduos indivíduos (457). Nenhum indivíduo ultrapassou 7,0 m (Fig. 1A). O diâmetro dos indivíduos inventariados variou de 2 a $14 \mathrm{~cm}$. Cerca de $42,9 \%$ dos indivíduos inventariados (273) apresentaram diâmetro entre 2 e $3 \mathrm{~cm}$ (Fig. 1B) e 540 indivíduos apresentaram até $5 \mathrm{~cm}$ diâm. $(84,8 \%)$. Em áreas de campo sujo, Ottmar et al. (2001) observaram que os indivíduos com diâmetro entre $2 \mathrm{e}$ $5 \mathrm{~cm}$ representam entre $71,7 \%$ e $89,4 \%$ do total de indivíduos inventariados. O número de indivíduos inventariados neste trabalho foi cerca de $50 \%$ superior ao apresentado por E.P. Rocha e Silva (dados não publicados) para uma área de 4 ha de campo sujo, o qual considerou apenas os indivíduos com diâmetro igual ou maior a $5 \mathrm{~cm}$.
Impacto das queimadas - Entre os 192 indivíduos que sofreram mortalidade aérea após a primeira queimada, 171 permaneceram neste estado após a segunda queimada e 21 morreram (12\%) (Tab. 2). Como conseqüência da terceira queimada, morreram cerca de $20 \%$ dos 171 indivíduos que permaneciam com mortalidade aérea após as duas queimadas. A importância deste tipo de dano pode ser avaliada se se considera que, após a segunda queimada, 39 indivíduos morreram, sendo que $57 \%$ destes haviam sofrido

Tabela 1. Lista de espécies, número de indivíduos e freqüência relativa (Fr) do inventário realizado em 1998, em área de campo sujo, protegida de queimada por 23 anos, na Reserva Ecológica do IBGE, DF, Brasil.

\begin{tabular}{|c|c|c|}
\hline Espécie & indivíduos & $\mathrm{Fr}$ \\
\hline Acosmium dasycarpum (Vogel) Yakovlev & 54 & 8,5 \\
\hline Andira paniculata Benth. & 4 & 0,6 \\
\hline Anonna crassiflora Mart. & 1 & 0,2 \\
\hline Aspidosperma tomentosum Mart. & 2 & 0,3 \\
\hline Byrsonima coccolobifolia Kunth. & 7 & 1,1 \\
\hline Byrsonima crassa Ndz. & 5 & 0,8 \\
\hline Byrsonima sp. & 1 & 0,2 \\
\hline Byrsonima verbascifolia (L.) Rich. & 28 & 4,4 \\
\hline Caryocar brasiliense Camb. & 2 & 0,3 \\
\hline Casearia sylvestris Sw. & 1 & 0,2 \\
\hline Connarus suberosus Planch. & 14 & 2,2 \\
\hline Dalbergia miscolobium Benth. & 1 & 0,2 \\
\hline Davilla elliptica A. St.-Hil. & 92 & 14,5 \\
\hline Dimorphandra mollis Benth. & 12 & 1,9 \\
\hline Enterolobium gummiferum (Mart.) J.F. Macbr. & 5 & 0,8 \\
\hline Eremanthus goyazensis (Gard.) Sch. Bip. & 37 & 5,8 \\
\hline Erythroxylum daphnites Mart. & 1 & 0,2 \\
\hline Erythroxylum deciduum A. St.-Hil. & 8 & 1,3 \\
\hline Erythroxylum suberosum A. St.-Hil. & 22 & 3,5 \\
\hline Erythroxylum tortuosum Mart. & 2 & 0,3 \\
\hline Hancornia speciosa Gomez & 1 & 0,2 \\
\hline Heteropterys byrsonimifolia A. Juss. & 2 & 0,3 \\
\hline Kielmeyera coriacea (Spreng.) Mart & 37 & 5,8 \\
\hline Miconia albicans (Sw.) Triana & 1 & 0,2 \\
\hline Neea theifera Oerst. & 6 & 0,9 \\
\hline Ouratea hexasperma Baill. & 38 & 6,0 \\
\hline Palicourea rigida H.B.K. & 7 & 1,1 \\
\hline Piptadenia sp. & 1 & 0,2 \\
\hline Psidium pohlianum Berg. & 1 & 0,2 \\
\hline Qualea grandiflora Mart. & 9 & 1,4 \\
\hline Qualea parviflora Mart. & 3 & 0,5 \\
\hline Roupala montana Aubl. & 123 & 19,3 \\
\hline Rourea induta Planch. & 20 & 3,1 \\
\hline Schefflrea macrocarpa (Seem.) D.C. Frodin & 4 & 0,7 \\
\hline Stryphnodendron adstringens (Mart.) Cov. & 4 & 0,6 \\
\hline Styrax ferrugineus Nees \& Mart. & 42 & 6,7 \\
\hline Symplocos rhamnifolia A. DC. & 4 & 0,6 \\
\hline Vellozia flavicans Mart. ex Schult. & 34 & 5,3 \\
\hline Total & 636 & 100,0 \\
\hline
\end{tabular}




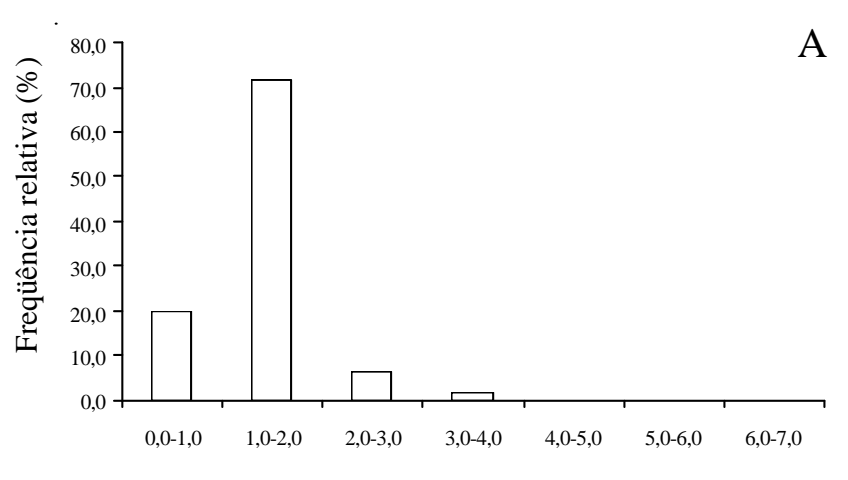

Classes de altura (m)

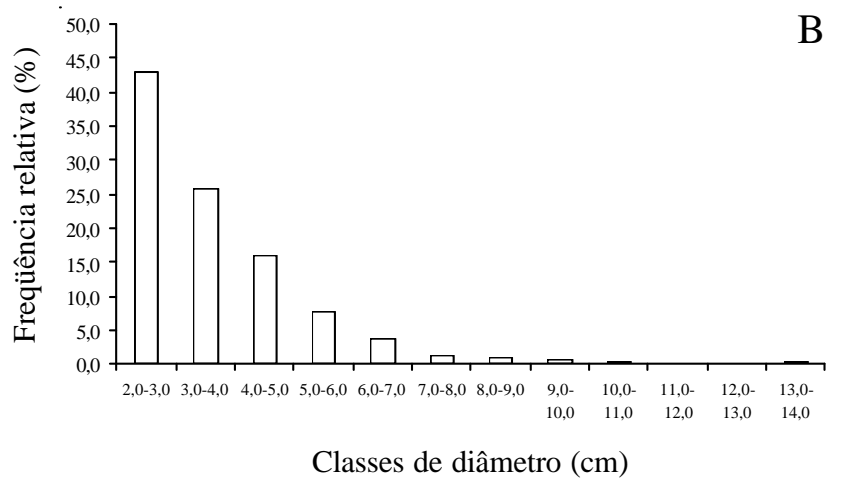

Figura 1. Freqüência relativa de altura (A) e diâmetro (B) dos indivíduos inventariados em área de campo sujo, em agosto/1998 (23 anos sem queima) na Reserva Ecológica do IBGE, DF, Brasil. $(\mathrm{N}=636)$.

mortalidade aérea na queimada anterior e que dos 52 indivíduos mortos na terceira queimada, $73 \%$ haviam sofrido mortalidade aérea ainda na queimada de 1998. Hoffmann \& Solbrig (2003) verificaram que fração considerável de indivíduos nas menores classes de diâmetro de algumas espécies lenhosas do Cerrado que sofrem mortalidade da parte aérea recuperou o tamanho pré-fogo dentro de um ano após a queima. Entretanto, para indivíduos maiores, esse tipo de dano representa perda considerável da parte vegetativa, a qual não é rapidamente recuperada. Nesse estudo, acima de $10 \%$ dos indivíduos com diâmetro superior a $5 \mathrm{~cm}$ sofreram mortalidade da parte aérea, nas três

Tabela 2. Número de indivíduos vivos, mortos e com mortalidade aérea em área de campo sujo, após três queimadas prescritas consecutivas, na Reserva Ecológica do IBGE, DF, Brasil.

\begin{tabular}{lccc}
\hline N. de indivíduos & $\begin{array}{c}\text { Primeira } \\
\text { queimada }\end{array}$ & $\begin{array}{c}\text { Segunda } \\
\text { queimada }\end{array}$ & $\begin{array}{c}\text { Terceira } \\
\text { queimanda }\end{array}$ \\
\hline Vivos & 636 & 493 & 454 \\
Mortos & 143 & 39 & 52 \\
Mortalidade aérea & 192 & 171 & 136 \\
\hline
\end{tabular}

queimadas. Mesmo indivíduos de maior porte podem sofrer este tipo de dano (Williams et al. 1999), tornando-os vulneráveis às queimadas freqüentes.

As taxas de mortalidade calculadas para as queimadas anuais (Equação 1), considerando o número de mortos na Tab. 2, foram de 22,5, 7,9 e 11,5\%, após as queimadas de 1998, 1999 e 2000 respectivamente, causando redução de cerca de $37 \%$ no número de indivíduos vivos. Os dados, principalmente da primeira queimada, são superiores aos apresentados por Sato et al. (1998) e E.P. Rocha e Silva (dados não publicados) para queimadas de cerrado sensu stricto e campo sujo. A elevada mortalidade calculada para a queimada de 1998 pode ser também conseqüência da inclusão no inventário de indivíduos com diâmetros menores que os considerados por outros autores. E.P. Rocha e Silva (dados não publicados) encontrou, para campo sujo, valores entre 5,2 a 9,8\% em regime bienal de fogo e, entre 9,7 e 12,3\% em um regime quadrienal, considerando, para o inventário, diâmetros superiores a $5 \mathrm{~cm}$. Sato et al. (1998) observaram taxas de mortalidade entre 6,4 e 13,0\% em áreas de cerrado sensu stricto, para indivíduos com diâmetro a partir de $5 \mathrm{~cm}$ no inventário.

O maior número de indivíduos mortos ocorreu na classe de altura entre 1 e $2 \mathrm{~m}$, sendo que os valores atingiram 98 indivíduos (freqüência relativa de 68,5\%), 33 indivíduos (freqüência relativa de 84,6\%) e 41 indivíduos (frequiência relativa de $78,7 \%$ ), após as queimadas de 1998, 1999 e 2000, respectivamente (Tab. 3). Não houve diferenças significativas entre as queimadas $\left(\chi^{2}=4,82, \mathrm{p}>0,05\right)$ quanto à proporção de indivíduos nas classes de altura. Apenas quatro indivíduos, nas classes de altura entre $2 \mathrm{~m}$ e $3 \mathrm{~m}$, morreram como consequência das três queimadas. Estes dados são similares aos encontrados por Sato et al. (1998) e E.P. Rocha e Silva (dados não publicados). De forma geral, durante queimadas, a mortalidade é maior em indivíduos de menor porte (Whelan 1995) por apresentarem cascas menos espessas e, em áreas com vegetação predominantemente graminosa, por estarem expostos à zona de maior temperatura durante a queimada (Frost \& Robertson 1987; Miranda et al. 1993; Miranda et al. 1996).

Houve maior percentagem de mortalidade nas classes de diâmetro menores, entre 2 e $3 \mathrm{~cm}$, com 84 indivíduos (frequiência relativa de 58,7\%), 20 indivíduos (freqüência relativa de $51,3 \%$ ) e 23 indivíduos (frequiência relativa de 44,2\%), após as queimadas de 1998, 1999 e 2000, respectivamente (Tab. 4). A classe 
Tabela 3. Número de indivíduos e frequiência relativa (Fr.) por classes de altura que sofreram mortalidade após três queimadas prescritas consecutivas, em área de campo sujo, na Reserva Ecológica do IBGE, DF, Brasil.

\begin{tabular}{|c|c|c|c|c|c|c|c|}
\hline \multirow{2}{*}{$\begin{array}{l}\text { Classes de altura } \\
\text { (m) }\end{array}$} & \multicolumn{2}{|c|}{ Primeira queimada } & \multicolumn{2}{|c|}{ Segunda queimada } & \multicolumn{2}{|c|}{ Terceira queimada } & \multirow{2}{*}{$\begin{array}{c}\text { Total } \\
\text { N. indivíduo }\end{array}$} \\
\hline & N. indivíduos & Fr. & N. indivíduos & Fr. & N. indivíduos & Fr. & \\
\hline $0-1,0$ & 42 & 29,4 & 6 & 15,4 & 10 & 19,8 & 58 \\
\hline $1,0-2,0$ & 98 & 68,5 & 33 & 84,6 & 41 & 78,7 & 172 \\
\hline $2,0-3,0$ & 3 & 2,1 & - & & 1 & 1,5 & 4 \\
\hline Total & 143 & 100 & 39 & 100 & 52 & 100 & 234 \\
\hline
\end{tabular}

de diâmetro entre 3 e $4 \mathrm{~cm}$ também concentrou grande número de indivíduos mortos, representando 34 indivíduos (frequiência relativa de 23,8\%), 9 indivíduos (frequêencia relativa de 23,1\%) e 18 indivíduos (freqüência relativa de $34,6 \%$ ) do total, após as queimadas de 1998, 1999 e 2000, respectivamente. Não houve diferenças significativas entre as queimadas $\left(\chi^{2}=32,81, p>0,05\right)$ quanto às proporções nas classes de diâmetro. E.P. Rocha \& Silva (dados não publicados) verificou maior mortalidade de indivíduos nas classes de diâmetro entre 5,0 e $5,5 \mathrm{~cm}$, após queimadas bienais e quadrienais em áreas de campo sujo e, em áreas de cerrado sensu stricto, Sato et al. (1998) observou resultados similares.

Os dados encontrados neste estudo mostram que o diâmetro dos indivíduos é fator determinante para a sobrevivência. Assim, mesmo plantas não muito altas podem sobreviver, caso o diâmetro do caule atinja um limite mínimo. Os dados obtidos também mostram que acima de $90 \%$ da mortalidade concentrou-se nas classes de diâmetro menores do que $5 \mathrm{~cm}$, tornando subestimados os valores apresentados na literatura que consideram este o diâmetro mínimo para inclusão em inventários para avaliação de impactos de queimadas na vegetação lenhosa do cerrado.
Ao se considerar apenas os indivíduos mortos que apresentavam diâmetro maior ou igual a $5 \mathrm{~cm}$, a mortalidade acumulada nas três queimadas anuais foi de $20,2 \%$, taxa semelhante à obtida por E.P. Rocha e Silva (dados não publicados) após três queimadas bienais $(20,9 \%)$ e duas quadrienais $(20,8 \%)$ em áreas de campo sujo, evidenciando que os indivíduos com diâmetro superior a $5 \mathrm{~cm}$ podem apresentar maior possibilidade de sobrevivência.

O elevado número de indivíduos com mortalidade da parte aérea pode ser consequiência do curto intervalo de tempo entre as queimadas, que não seria suficiente para o espessamento da casca nas rebrotas ou para o seu crescimento além da zona de chamas. A maior proporção destes indivíduos concentrou-se na classe de altura entre 1,0 e 1,5 m, com 119 indivíduos (freqüência relativa de 61,3\%), 33 indivíduos (frequiência relativa de 50,0\%) e 48 indivíduos (frequiência relativa de $48,4 \%$ ) após as queimadas de 1998, 1999 e 2000, respectivamente (Tab. 5). Não houve diferenças significativas entre as queimadas $\left(\chi^{2}=14,29, p>0,05\right)$ quanto às proporções de indivíduos nas classes de altura. Dados semelhantes foram encontrados por E.P. Rocha e Silva (dados não publicados) para campo sujo e por Norton-Griffiths

Tabela 4. Número de indivíduos e freqüência relativa (Fr.) por classes de diâmetro que sofreram mortalidade após três queimadas prescritas consecutivas, em área de campo sujo, na Reserva Ecológica do IBGE, DF, Brasil.

\begin{tabular}{|c|c|c|c|c|c|c|c|}
\hline \multirow{2}{*}{$\begin{array}{l}\text { Classes de diâmetro } \\
(\mathrm{cm})\end{array}$} & \multicolumn{2}{|c|}{ Primeira queimada } & \multicolumn{2}{|c|}{ Segunda queimada } & \multicolumn{2}{|c|}{ Terceira queimada } & \multirow{2}{*}{$\begin{array}{c}\text { Total } \\
\text { N. indivíduos }\end{array}$} \\
\hline & N. indivíduos & Fr. & N. indivíduos & Fr. & N. indivíduos & Fr. & \\
\hline $2,0-3,0$ & 84 & 58,7 & 20 & 51,3 & 23 & 45,2 & 127 \\
\hline $3,0-4,0$ & 34 & 23,8 & 9 & 23,1 & 18 & 34,6 & 61 \\
\hline $4,0-5,0$ & 17 & 11,9 & 7 & 17,9 & 5 & 10,5 & 29 \\
\hline $5,0-6,0$ & 7 & 4,9 & 1 & 2,6 & 3 & 5,0 & 11 \\
\hline $6,0-7,0$ & - & 0 & 2 & 5,1 & 2 & 3,2 & 4 \\
\hline $7,0-8,0$ & - & 0 & - & - & 1 & 1,5 & 1 \\
\hline $8,0-9,0$ & 1 & 0,7 & - & - & - & - & 1 \\
\hline Total & 143 & 100 & 39 & 100 & 52 & 100 & 234 \\
\hline
\end{tabular}


(1984), que registrou, em savanas africanas, maior percentagem de mortalidade da parte aérea para indivíduos com menos de $1 \mathrm{~m}$ alt.

Os dados mostraram que os indivíduos nas classes menores de diâmetro, até $5 \mathrm{~cm}$, foram mais afetados com mortalidade aérea, com 181 indivíduos (freqüência relativa de 86,6\%), 62 indivíduos (freqüência relativa de $93,9 \%$ ) e 94 indivíduos (freqüência relativa de 95,5\%) após as queimadas de 1998, 1999 e 2000, respectivamente (Tab. 6). Não houve diferenças significativas entre as queimadas $\left.\chi^{2}=4,48, p>0,05\right)$ quanto às proporções de indivíduos nas classes de diâmetro. A elevada proporção de mortalidade aérea nestas classes de diâmetro reflete a predominância de indivíduos nestas classes no inventário inicial, assim como sua baixa tolerância à ocorrência de queimadas. E.P. Rocha e Silva (dados não publicados) não encontrou relação entre mortalidade aérea e diâmetro. Porém, Hoffmann \& Solbrig (2003) verificaram elevada incidência de mortalidade aérea em indivíduos com menores diâmetros.

A redução na parte vegetativa dessas plantas devido à elevada incidência de mortalidade aérea deve impactar de modo significativo o crescimento das populações, como observado em outros estudos (Hoffmann 1998; 1999). Entretanto, a elevada incidência de mortalidade da parte aérea deve impactar em maior extensão as espécies arbóreas do que arbustos e sub-arbustos, considerando que estes necessitam de tempo menor para alcançar a maturidade sexual (Hoffmann \& Solbrig 2003). Assim, várias espécies de arbustos e sub-arbustos no Cerrado são mais favorecidas por queimadas freqüentes, em comparação com as espécies de árvores (Moreira 2000).

As três queimadas anuais resultaram na morte de cerca de $36 \%$ dos indivíduos, indicando alteração significativa na fisionomia da área. Além disso, os caules destruídos incluíram $73 \%$ dos indivíduos, reforçando a importância deste tipo de dano para a estrutura e funcionamento do sistema. Hoffmann (1999) considera que, apesar do impacto reduzido em indivíduos estabelecidos, o fogo deve ter impacto negativo forte no crescimento populacional de várias espécies lenhosas do Cerrado.

Com os dados obtidos neste estudo, são reforçadas as considerações de forte impacto negativo na estrutura

Tabela 5. Número de indivíduos e freqüência relativa (Fr.) em diferentes classes de altura que sofreram mortalidade da parte aérea ao longo de três queimadas prescritas anuais, em área de campo sujo, na Reserva Ecológica do IBGE, DF, Brasil.

\begin{tabular}{|c|c|c|c|c|c|c|c|}
\hline \multirow{2}{*}{$\begin{array}{l}\text { Classes de altura } \\
\qquad \text { (m) }\end{array}$} & \multicolumn{2}{|c|}{ Primeira queimada } & \multicolumn{2}{|c|}{ Segunda queimada } & \multicolumn{2}{|c|}{ Terceira queimada } & \multirow{2}{*}{$\begin{array}{c}\text { Total } \\
\text { N. indivíduos }\end{array}$} \\
\hline & N. indivíduos & Fr. & N. indivíduos & Fr. & N. indivíduos & Fr. & \\
\hline $0-0,5$ & - & - & 1 & 1,5 & - & - & 1 \\
\hline $0,5-1,0$ & 36 & 18,6 & 22 & 33,3 & 32 & 32,6 & 90 \\
\hline $1,0-1,5$ & 119 & 61,3 & 33 & 50,0 & 48 & 48,6 & 200 \\
\hline $1,5-2,0$ & 34 & 17,5 & 9 & 13,7 & 15 & 14,8 & 58 \\
\hline $2,0-2,5$ & 5 & 2,6 & 1 & 1,5 & 4 & 4,0 & 10 \\
\hline Total & 194 & 100 & 66 & 100 & 99 & 100 & 359 \\
\hline
\end{tabular}

Tabela 6. Número de indivíduos em diferentes classes de diâmetro que sofreram mortalidade da parte aérea ao longo de três queimadas prescritas anuais, em área de campo sujo, na Reserva Ecológica do IBGE, DF, Brasil.

\begin{tabular}{|c|c|c|c|c|c|c|c|}
\hline \multirow{2}{*}{$\begin{array}{c}\text { Classes de diâmetro } \\
(\mathrm{cm})\end{array}$} & \multicolumn{2}{|c|}{ Primeira queimada } & \multicolumn{2}{|c|}{ Segunda queimada } & \multicolumn{2}{|c|}{ Terceira queimada } & \multirow{2}{*}{$\begin{array}{c}\text { Total } \\
\text { N. indivíduos }\end{array}$} \\
\hline & N. indivíduos & Fr. & N. indivíduos & Fr. & N. indivíduos & Fr. & \\
\hline $2,0-3,0$ & 111 & 57,2 & 41 & 62,1 & 56 & 56,9 & 208 \\
\hline $3,0-4,0$ & 57 & 29,5 & 16 & 24,2 & 33 & 33,5 & 106 \\
\hline $4,0-5,0$ & 13 & 6,7 & 5 & 7,6 & 5 & 5,1 & 23 \\
\hline $5,0-6,0$ & 7 & 3,6 & 4 & 6,1 & 3 & 3,4 & 17 \\
\hline $6,0-7,0$ & 3 & 1,5 & - & - & 1 & 0,7 & 4 \\
\hline $7,0-8,0$ & - & - & - & - & - & - & - \\
\hline $8,0-9,0$ & 2 & 1 & - & - & - & - & 3 \\
\hline $9,0-10,0$ & 1 & 0,5 & - & - & 1 & 0,4 & 2 \\
\hline Total & 194 & 100 & 66 & 100 & 99 & 100 & 363 \\
\hline
\end{tabular}


da comunidade de lenhosas de campo sujo em queimadas anuais, as quais simulam o atual regime de fogo com elevada freqüência para o Cerrado. Os resultados encontrados, com taxas de mortalidade elevadas, também demonstram maior amplitude destes danos, em comparação com estudos anteriores.

\section{Agradecimentos}

Os autores agradecem aos Drs. Manoel Cláudio Silva Júnior, John DuVall Hay, Helena Castanheira de Morais, Bráulio Souza Dias, William Hoffmann, Mundayatan Haridassan e José Felipe Ribeiro, pelas sugestões e comentários apresentados durante o trabalho; aos colegas e funcionários do Laboratório de Ecologia da Universidade de Brasília Pedro Olavo, Antônio Gumiero e Naomi Sato, pelo auxílio nos trabalhos de campo; à Maria Iracema Gonzáles, Chefe da Divisão de Estudos Ambientais do Cerrado da Reserva Ecológica do IBGE, pela licença de pesquisa na área; ao técnico Diassis Alvarenga e à Brigada de Combate a Incêndios do IBGE, pelo apoio no campo e ao Serviço Florestal do Departamento de Agricultura dos Estados Unidos (USDA/FS); ao Conselho Nacional de Desenvolvimento Científico e Tecnológico (CNPq), através do Programa de Pesquisas Ecológicas de Longa Duração (PELD), pelo apoio financeiro.

\section{Referências bibliográficas}

Agee, J.K. 1993. Fire Ecology of Pacific Northwest Forests. New York, Island Press.

Bond, W.J. \& Wilgen, B.W. 1996. Fire and Plants. New York, Chapman \& Hall.

Coutinho, L.M. 1990. Fire in the ecology of the Brazilian cerrado. Pp. 82-105. In: J.G. Goldammer (ed.). Fire in the Tropical Biota. Berlin, Springer-Verlag.

Eiten, G. 1994. Vegetação do Cerrado. Pp. 17-74. In: M.N. Pinto (org.). Cerrado: caracterização, ocupação e perspectivas. Brasília, Editora Universidade de Brasília.

Franco, A.C.; Souza, M.P. \& Nardoto, G.B. 1996. Estabelecimento e crescimento de Dalbergia miscolobium em áreas de campo sujo e cerrado no DF. Pp. 84-92. In: H.S. Miranda; B.F.S. Dias \& C.H. Saito (orgs.). Impacto de Queimadas em Área de Cerrado e Restinga. Brasília, ECL/Universidade de Brasília.

Frost, P.G.H. \& Robertson, F. 1987. The ecological effects of fire in savannas. Pp. 93-140. In: B.H. Walker (ed.). Determinants of Tropical Savannas. Oxford, IRL Press. Hoffmann, W.A. 1998. Post-burn reproduction of woody plants in a neotropical savanna: the relative importance of sexual and vegetative reproduction. Journal of Applied Ecology 35: 422-433.
Hoffmann, W.A. 1999. Fire and population dynamics of woody plants in a neotropical savanna: matrix model projections. Ecology 80: 1354-1369.

Hoffmann, W.A. \& Solbrig, O.T. 2003. The role of topkill in the differential response of savanna woody species to fire. Forest Ecology and Management 180: 273-286.

Landim, M.F. \& Hay, J.D. 1995. Impacto do fogo sobre alguns aspectos da biologia reprodutiva de Kielmeyera coriacea Mart. Revista Brasileira de Biologia 56(1): 127-134.

Medina, E. \& Silva, J.F. 1990. Savannas of northern South América: a steady state regulated by water-fire interactions on a background of low nutrient availability. Journal of Biogeography 17: 403-413.

Medina, E. \& Huber, O. 1994. The role of biodiversity in the functioning of savanna ecosystems. In: O.T. Solbrig; H.M. Emden \& P.G.W.J. Oordt (eds.). Biodiversity and Global Change. IUBS. Chicago, CAB International.

Miranda, A.C.; Miranda, H.S.; Dias, I.F.O. \& Dias, B.F.S. 1993. Soil and air temperatures during prescribed Cerrado fires in Central Brazil. Journal of Tropical Ecology 9: 313-320.

Miranda, H.S.; Rocha e Silva, E.P. \& Miranda, A.C. 1996. Comportamento do fogo em queimadas de campo sujo. Pp. 1-10. In: H.S. Miranda; B.F.S. Dias \& C.H. Saito (orgs.). Impacto de Queimadas em Área de Cerrado e Restinga. Brasília, ECL/Universidade de Brasília.

Moreira, A.G. 2000. Effects of fire protection on savanna structure in central Brazil. Journal of Biogeography 27: 1021-1029.

Norton-Griffths, M. 1984. The influence of grazing, browsing and fire on the vegetation dynamics of the Serengeti. Pp. 310-352. In: A.R.E. Sinclair \& M. Norton-Griffths (eds.). Serengeti - Dynamics of an Ecosystem. Chicago, University of Chicago.

Ottmar, R.D.; Vihnanek, R.E.; Miranda, H.S.; Sato, M.N. \& Andrade, S.M.A. 2001. Séries de estéreo-fotografias para quantificar a biomassa da vegetação do cerrado do Brasil Central. v.I. USDA. General Technical Report. PNW GTR 5191.

Rawitscher, F.K.; Ferri, M.G. \& Rachid, M. 1943. Profundidade dos solos e vegetação em campos cerrados do Brasil Meridional. Anais da Academia Brasileira de Ciências 4: 267-294.

Rocha e Silva, E.P. \& Miranda, H.S. 1996. Temperatura do câmbio de espécies lenhosas do cerrado durante queimadas prescritas. Pp. 253-257. In: R.C. Pereira \& L.C.B. Nasser (eds.). Anais do VII Simpósio sobre o Cerrado. Brasília, EMBRAPA-CPAC.

Sato, M.N.; Garda, A.A. \& Miranda, H.S. 1998. Fire effects in the mortality rate of woody vegetation in Central Brazil. Pp. 1777-1784. In: D.X. Viegas (ed.). Proceedings of the $3^{\text {rd }}$ International Conference on Forest Fires Research. Coimbra.

Silva, G.T.; Sato, M.N. \& Miranda, H.S. 1996. Mortalidade de plantas lenhosas em um campo sujo de cerrado submetido a queimadas prescritas. Pp. 93-101. In: H.S. Miranda; B.F.S. Dias \& C.H. Saito (orgs.). Impacto de Queimadas em Área de Cerrado e Restinga. Brasília, ECL/Universidade de Brasília. 
500 Medeiros \& Miranda: Mortalidade pós-fogo em espécies lenhosas de campo sujo submetido a três queimadas...

Sokal, R.R. \& Rohlf, F.J. 1987. Biometry - the principles and practice of statistics in biological research. New York, W.H. Freeman \& Company.

Souza, M.H.A.O. \& Soares, J.J. 1983. Brotamento de espécies arbustivas e arbóreas posteriormente a uma queimada, num cerradão. Pp. 263-275. In: Anais do III Seminário Regional de Ecologia. São Carlos.
Statistica. 2000. Computer Software. Tulsa, Statsoft, Inc. Whelan, R.J. 1995. The Ecology of Fire. Cambridge, Cambridge University Press.

Williams, R.J.; Cook, G.D.; Gill, A.M. \& Moore, P.H.R. 1999. Fire regime, fire intensity and tree survival in a tropical savanna in northern Australia. Australian Journal of Ecology 24: 50-59. 